\title{
Experimental and Theoretical Study of the Effect of Functionalized Pyrene Polymerization on Carbon Electrode Surfaces for Electrochemical Storage
}

Dr. Bihag Anothumakkool, ${ }^{[\mathrm{a}, \mathrm{b},+]}$ Dr. Yuman Sayed-Ahmad-Baraza, ${ }^{[\mathrm{a},+]}$ Dr. Florian Massuyeau, ${ }^{[\mathrm{a}]}$ Prof. Thierry Brousse,${ }^{[\mathrm{a}, \mathrm{c}]}$ Dr. Chris Ewels, ${ }^{*[\mathrm{a}]}$ and Dr. Joel Gaubicher* ${ }^{[\mathrm{a}]}$

[a] Institut des Matériaux Jean Rouxel (IMN), University of Nantes, CNRS, 2, rue de Houssiniere-B.P. 32229-44322 Nantes cedex 3, France

E-mail: joel.gaubicher@cnrs-imn.fr

E-mail: chris.ewels@cnrs-imn.fr

[b] TNO-Holst Centre, High Tech Campus 31, 5605 KN Eindhoven, The Netherlands

[c] Réseau sur le Stockage Electrochimique de l'Energie (RS2E), FR CNRS 3459, France

$+\quad$ These authors equally contributed to this work

* $\quad$ Corresponding authors

Supporting information for this article is available on the WWW under http://dx.doi.org/10.1002/batt.202000329

An invited contribution to a Special Collection dedicated to Metal-Ion Hybrid Supercapacitors

Keywords : adsorption mechanism, electropolymerization mechanism, pyrene density functional theory, Li metal hybrid supercapacitor

Looking for the perfect match: The effect of functional groups on pyrene adsorption and related electropolymerization mechanisms on MWCNT model electrodes, as well as resulting electrochemical charge-storage properties in a Li metal supercapacitor configuration are reported based on experiment and DFT calculations. Trends of predicted ionization energies (HOMO levels) and Hammet substituent constants generally match with (measured) oxidation potentials of self-adsorbed monomers. Fusion rather than linear-type oligomerization takes 
place. Optimization of functional group and electrode loading allows 5-fold capacity increase thanks to p-doping of oligomers.

Gaubicher, Ewels (@ chrisewels), \& co-workers (@CNRS, @EnergyRS2E) show careful choice of functionalized pyrene additive to carbon electrodes allows 5-fold capacity increase for Li metal \#supercapacitors

\begin{abstract}
We report the effect of functional groups on pyrene adsorption and associated electropolymerization mechanisms on carbon nanotube model electrodes, as well as resulting electrochemical charge-storage properties in a Li metal supercapacitor configuration. The impact of pyrene functional groups is tested by varying the alkyl chain length as well as the composite electrode formulation protocol. From experiment and DFT calculations we conclude that (i) all pyrene derivatives bind strongly to the MWCNT surface with submonolayer loadings. Intermolecular forces and/or packing configurations rather than charge transfer between MWCNT and substrate likely play a major role in the extent of selfadsorption process (ii) trends of predicted ionization energies (-HOMO levels) and Hammet substituent constants match with (measured) oxidation potentials of adsorbed monomers. However, this correlation fails for unsubstitued pyrene presumably because of molecular aggregation, an argument supported by excimer formation in supernatent solutions, (iii) fusion rather than linear-type oligomerization takes place and is described for the first time and, (iv) longer alkyl chains lead to more extended functionalized oligomers which in turn enhances pdoping (reversible capacity). In a Li metal hybrid supercapacitor configuration, best capacitypower compromise was found with $55^{\wedge \wedge} \mathrm{wt} \%$ pyrene- $\left(\mathrm{CH}_{2}\right) \mathrm{COOH}$, resulting in a 5-fold capacity increase from $13^{\wedge \wedge} \mathrm{mAh} / \mathrm{g}_{\text {electrode }}\left(19^{\wedge \wedge} \mathrm{F} / \mathrm{g}_{\text {electrode }}\right)$ for untreated MWCNT to $68^{\wedge \wedge} \mathrm{mAh} / \mathrm{g}_{\text {electrode }}\left(102^{\wedge \wedge} \mathrm{F} / \mathrm{g}_{\text {electrode }}\right)$ between $2--4.4^{\wedge \wedge} \mathrm{V}$ vs. $\mathrm{Li}^{+} / \mathrm{Li}$.
\end{abstract}

\title{
1. Introduction
}

Efficient electrochemical energy storage devices are critical for stationary and portable applications. Among these $\mathrm{Li}^{[}{ }^{[1]}$ and Na-ion batteries, ${ }^{[2]}$ supercapacitors, ${ }^{[3]}$ and their hybrid 
systems ${ }^{[4]}$ are prominent due to their high energy and power density. The negative electrode/anode of such batteries and hybrid capacitors are already well advanced due to the availability of $\mathrm{Li}$ and $\mathrm{Na}$ chemical storage approaches ${ }^{[1,2]}$ with capacities greater than graphite. Associated success in tackling the first irreversible capacity of such new generation anode materials ${ }^{[5]}$ means they are close to commercialization. Thus the real bottleneck of Liion and Na-ion batteries, as well as their related hybrid technologies, is currently that benchmarked cathode materials all have relatively low theoretical capacities. From layered metal oxides (e.^g., $\mathrm{LiCoO}_{2}$ or $\mathrm{NaCoO}_{2}$ ) to new materials such as mixed layered, e.^g., $\mathrm{Li}$ (or $\mathrm{Na}) \mathrm{Ni}_{x} \mathrm{Mn}_{y} \mathrm{CozO}_{2}$ ) and conversion type materials, ${ }^{[6,7]}$ prevailing issues are poor power capability and capacity fading with cycling. In contrast the cycling stability is excellent in non-faradic storage, such as electrochemical capacitors (so-called supercapacitors), and Metal-ion hybrid capacitors which utilize capacitive-type double layer charge storage at the positive electrode usually with the help of high surface area carbons,.$^{[8-10]}$ However, this advantage is mitigated by their intrinsically lower energy density. ${ }^{[11]}$

An efficient approach to address this issue consists in coupling high capacity-high potential electroactive organic materials to electric double layer capacitor materials such as carbon upon molecular functionalization. One strategy consists in using $\pi-\pi$ stacking, ${ }^{[12-16]}$ of electroactive molecules at the surface of graphitic carbon materials, thereby providing additional faradic storage without damaging the underlying $\mathrm{sp}^{2}$-carbon domains. Polycyclic aromatic hydrocarbon molecules (PAHs) are an appropriate choice for this purpose due to their propensity to $\pi-\pi$ stack on graphitic surfaces. ${ }^{[17]}$ Waltman and Bargon ${ }^{[18]}$ extensively studied the electropolymerization of polycyclic hydrocarbon molecules at the surface of metal electrodes suggesting their possible application for energy storage, as they show very stable capacity during cycling. Several PAHs have been proposed for this purpose such as pyrene, indole and carbazole derivatives, ${ }^{[18--20]}$ Our group has shown the pyrene family displays excellent cycle life even at high positive potential, ${ }^{[12,21,22]}$ while electron-withdrawing 
substituents attached to pyrene enhance $\pi$-stacking type interactions with graphitic carbon'. ${ }^{[21,22]}$ Lee $e t^{\wedge \wedge} a{ }^{[12]}$ reported charge storage properties of carbon nanotube (MWCNT) composites with pyrene functionalized with $\left\langle\mathrm{C}->\mathrm{NH}_{2}\right.$ and $\langle\mathrm{C}->\mathrm{COOH}$ groups. Interestingly, proton release accompanying the formation of the pyrene radical on oxidation can also be used in LICs or NICs to prelithiate(sodate) the negative electrode ${ }^{[23]}$ which is one of the persisting key challenges.

Here, we investigate the effect of attaching a polar group $\left(<\mathrm{C}->\left(\mathrm{CH}_{2}\right)_{n}<\mathrm{C}->\mathrm{COOH}\right)$ $(n=0,1,3)$ to a pyrene moiety, not only on the $\pi-\pi$ interaction with the graphitic carbon surface but also on the electropolymerization process, findings being interpreted with the aid of DFT calculations. Based on these results, the storage capability of the resulting electrode is then determined in a lithium metal hybrid configuration.

\section{Experimental Section}

\section{Materials}

Pyrene (Pyr), 1-pyrenecarboxylic acid (PAc), 1-pyreneacetic acid (PMeAc) and 1pyrenebutyric acid (PPrAc) were purchased from Aldrich Chemicals. $1^{\wedge \wedge} \mathrm{M} \mathrm{LiPF}$ in $1^{\wedge}: \wedge 1^{\wedge \wedge} \mathrm{Wt} \%$ ethelenecarbonate/dimethylcarbonate (LP-30) was purchased from BASF, multi-walled carbon nanotubes came from Showa Denko (VGCF-X). Carbon Super P® (Csp) conductive carbon black was purchased from TIMCAL. Activated carbon, YP-80F was purchased from Kuraraychemical, PvDF binders from Kynar® and $N$-methylpyrrolidone (NMP) were purchased from Aldrich.

\section{Positive electrode preparation}

Three methods were used to prepare the MWCNT-Pyrene composites. In the first, referred to as Protocol 1, MWCNT electrode $\left(20^{\wedge \wedge} \mathrm{mg} / \mathrm{cm}^{2}\right)$ films pressed between stainless steel meshes were soaked in $4^{\wedge \wedge} \mathrm{mM}$ pyrene molecules in ethanol for $3^{\wedge \wedge}$ days followed by washing with ethanol to remove any non-adsorbed molecules. These composites of pyrene, 1-pyrenecarboxylic acid, 1- 
pyreneacetic acid and 1-pyrenebutyric acid are denoted $\mathrm{C}_{\mathrm{ad}} \mathrm{Pyr}, \mathrm{C}_{\mathrm{ad}} \mathrm{PrAc}, \mathrm{C}_{\mathrm{ad}} \mathrm{PMe} A \mathrm{c}$ and $\mathrm{C}_{\mathrm{ad}} \mathrm{PPrAc}$ respectively. For the sake of comparison, a second method, referred to as Protocol 2, was used. This consists of in-situ electropolymerization of 1-pyrenebutyric acid, by cycling a MWCNT electrode at a scan rate of $5^{\wedge \wedge} \mathrm{mV} / \mathrm{s}$ in $7^{\wedge \wedge} \mathrm{ml}$ of LP-30 containing $2.5^{\wedge \wedge} \mathrm{mM} 1$-pyrenebutyric acid using a threeelectrode cell. Finally, we introduce a third protocol referred to as Protocol 3, in which electrode ink was prepared by mixing the MWCNT, pyrene molecules in NMP and PVdF $\left(5^{\wedge \wedge} \mathrm{Wt} \%\right)$ followed by ball milling for $30^{\wedge \wedge}$ minutes at a speed of $600^{\wedge \wedge} \mathrm{rpm}$. The ink was then roll-coated on $\mathrm{Al}$ foil and dried $\left(1^{\wedge \wedge} \mathrm{mg} / \mathrm{cm}^{2}\right)$. These composite electrodes of pyrene, 1-pyrenecarboxylic acid, 1-pyreneacetic acid and 1-pyrenebutyric acid are denoted as CPyr, CPAc, CPMeAc and CPPrAc respectively. Similarly, 1pyreneacetic acid was combined with YP-80 and is denoted YP-PMeAc. The last numbers in the acronym indicate the $\mathrm{wt}^{\wedge} \%$ of pyrene molecules in the composite.

\section{Physical characterizations}

The weight percentage $\left(\mathrm{wt}^{\wedge} \%\right)$ of molecules in the composites was derived from TGA measurements at $5^{\wedge \wedge} \mathrm{K} / \mathrm{min}$ under $\mathrm{N}_{2}$ atmosphere from room temperature to $900^{\wedge}{ }^{\circ} \mathrm{C}$ using Perkin Elmer TGS2 apparatus. Scanning electron microscopy (SEM) was carried out using a Jeol JSM-7600F microscope. Infrared spectra were obtained in ATR mode using a Bruker ALPHA placed inside the same glove box as the one used for battery assembly. Photoluminescence emission (PL) spectra were carried out on a Horiba Jobin-Yvon Flurolog 3 equipped with a $450^{\wedge \wedge} \mathrm{W}$ xenon lamp by means of a R13456 PMT detector from Hamamatsu. PL spectra were obtained with an excitation of $345^{\wedge \wedge} \mathrm{nm}$ in liquid solution in front face position.

\section{Electrochemical characterization}

A modified Swagelok cell with a dimension of $12^{\wedge \wedge} \mathrm{mm}$ was used for electrochemical measurements. Electrodes were cut into the specific dimension and kept between glass-fiber separators soaked in LP-30 as the electrolyte. Cell assembly was carried out in a moisture free glove box 
$\left(\mathrm{H}_{2} \mathrm{O}<1^{\wedge \wedge} \mathrm{ppm}, \mathrm{O}_{2}<0.3^{\wedge \wedge} \mathrm{ppm}\right)$. All electrochemical measurements were monitored in Bio-Logic VMP-2 or -3. Reference and negative electrodes were Li metal and all the potentials are reported against a $\mathrm{Li}^{+} / \mathrm{Li}$. Electropolymerization was performed in a potential window of $2--4.4^{\wedge \wedge} \mathrm{V} \mathrm{vs}$. $\mathrm{Li}^{+} / \mathrm{Li}$ at a constant current of $0.1^{\wedge \wedge} \mathrm{A} / \mathrm{g}$. Electrochemical impedance (EIS) analysis was conducted using a sinusoidal potential perturbation with a $10^{\wedge \wedge} \mathrm{mV}\left(V_{\mathrm{rms}}=7.07^{\wedge \wedge} \mathrm{mV}\right)$ amplitude from $100^{\wedge \wedge} \mathrm{kHz}$ to $0.01^{\wedge \wedge} \mathrm{Hz}$

\section{Computational modelling}

Density Functional Theory (DFT) calculations under the local density approximation (LDA) were performed using the AIMPRO code.$^{[24,25,26]}$ For modelling the interaction between the molecules and the CNTs, pyrene and its derivatives were placed on large 200 -atom hexagonal periodic $10 \times 10$ graphene cells, with an in-plane lattice constant of $24.43^{\wedge \wedge} \AA$. Graphene is a reasonable approximation for these large MWCNTs where exterior surface curvature effects are negligible. In addition, the work function of MWCNTs with sizes comparable to those used here is theoretically expected to be very similar to graphene. ${ }^{[27]}$ Interlayer spacing was set to over $28^{\wedge \wedge} \AA$ to avoid interaction between neighboring sheets. The isolated molecules were modelled in hexagonal cells the same size as the simulations incorporating graphene.

Charge density was constructed on a real-space grid with an energy cut-off of $150^{\wedge \wedge} \mathrm{Ha}$ $\left(200^{\wedge \wedge} \mathrm{Ha}\right.$ when oxygen present), while Kohn-Sham wave functions were constructed using localized Gaussian-based orbital functions (38/12/40 for C/H/O respectively, up to maximum angular momentum $l=2$ ). Relativistic pseudopotentials generated by Hartwigsen, Goedecker and Hutter ${ }^{[28]}$ were used, leaving 4, 1 and 6 non-core electrons for $\mathrm{C}, \mathrm{H}$ and $\mathrm{O}$ respectively.

The self-consistency cycle was considered to be converged when the change in the absolute energy was less than $10^{<\mathrm{M}->7 \wedge \wedge} \mathrm{Ha}$, except for the calculations (optimization and Mulliken analysis) involved in the study of partial atomic charges and spin densities of the neutral and charged isolated molecules presented in Figure ${ }^{\wedge \wedge} 6<x$ figr6>, where the tolerance was set at $10^{<\mathrm{M}->5 \wedge \wedge} \mathrm{Ha}$. All atom 
positions were fully optimized, using a conjugate gradient algorithm until the maximum atomic position change in a given iteration dropped below $10^{<M->4} \mathrm{a}_{0}\left(\mathrm{a}_{0}\right.$ : Bohr radius) and/or the energy change was less than $10^{<\mathrm{M}->5 \wedge \wedge} \mathrm{Ha}$, while lattice constants were fixed. For the +1 cations spin-polarized calculations were performed with system spin allowed to vary freely, in all cases giving final cation spin states with one unpaired electron. Atomic charge states were calculated using Mulliken population analysis. Since absolute Mulliken charges are known to be often inaccurate we focus here on relative changes in Mulliken charge values.

A $3 \times 3 \times 1 k$-point grid centered at $\Gamma$ under the Monkhorst and Pack scheme ${ }^{[29]}$ was used for integrating the Brillouin zone in the models involving graphene, while a single $k$-point was used for the calculations involving isolated molecules. Density of states (DOS) and partial density of states (PDOS) plots for the functionalized graphene models use a $\Gamma$-centered $k$-point grid of $6 \times 6 \times 1$ for the electronic density and $36 \times 36 \times 1$ to generate the DOS/PDOS, with sequential Gaussian broadenings of $10^{\wedge \wedge} \mathrm{meV}$ and $80^{\wedge \wedge} \mathrm{meV}$ full width at half maximum (FWHM) applied for the plots. Projection of the Kohn-Sham states for the PDOS uses Mulliken populations. Electronic levels are quoted after alignment of the vacuum level to zero. Alignment of the vacuum level is performed by computing the averaged electrostatic potential over the cross-section perpendicular to the molecular/graphene plane, and subtracting its average value over the vacuum slab region from the energy of the electronic levels. Binding energies of pyrene and its derivatives with graphene are calculated by subtracting the total energy of the isolated free molecule and graphene models from the total energy of the corresponding functionalized graphene system. In all cases the same molecular conformation is considered for the corresponding free and functionalized states.

We note that all DFT calculations were performed on model graphene surfaces whereas experiments used MWCNTs. A complete discussion reported in supplementary information indicates that any errors coming from the differences between our graphene model and a more realistic MWCNT model are most likely very small compared to other effects such as local variations in 
stacking and packing density, possible presence of defects and impurities formed during growth process (amorphous carbon, metallic catalyst, polyaromatic surface material, solvent, surfactants, etc.).

In order to estimate the molecular density of an adsorbed monolayer of pyrene associated with a specific molecular arrangement, an approximate "Van der Waals surface area" for each molecule is calculated (Figure ${ }^{\wedge} \mathrm{S} 1$ describes how this is done). This gives a pyrene "Van der Waals area" of $6.79 \times 10^{5 \wedge \wedge} \mathrm{pm}^{2}$ for planar (surface parallel), $3.57 \times 10^{5 \wedge \wedge} \mathrm{pm}^{2}$ for lateral (surface perpendicular, molecular long axis parallel to surface) and $2.75 \times 10^{5 \wedge \wedge} \mathrm{pm}^{2}$ for vertical (surface perpendicular, molecular long axis perpendicular to surface) configurations. These values correspond to molar surface densities of $2.45 \times 10^{<\mathrm{M}->10 \wedge \wedge} \mathrm{mol} / \mathrm{cm}^{2}, 4.66 \times 10^{<\mathrm{M}->10 \wedge \wedge} \mathrm{mol} / \mathrm{cm}^{2}$ and $6.04 \times 10^{<\mathrm{M}->10 \wedge \wedge} \mathrm{mol} / \mathrm{cm}^{2}$ respectively for an ideal fully-packed monolayer, and can be considered as limit values.

\section{Results and Discussion}

Representation of the series of pyrene derivatives as well as SEM image of multiwall carbon nanotubes (MWCNT) substrate used in this study are shown in Figure ${ }^{\wedge \wedge} 1<$ figr $1>a--b$ respectively. Comparison of PMeAc and PMeALi FTIR spectra (Figure ${ }^{\wedge} \mathrm{S} 2$ ) confirm the disapearance of the PMeAc carbonyl bond at $1710^{\wedge \wedge} \mathrm{cm}^{<\mathrm{M}->1}$. The specific surface area (SSA) of the current MWCNTs is $294^{\wedge} \wedge \mathrm{m}^{2} / \mathrm{g}$, most of it corresponding to "open" surface (accessible to both ions and molecules). The amount of self-adsorbed molecules (loading) on the MWCNT surfaces was first evaluated for pure pyrene (Pyr) and compared to that obtained according to the number $n$ of $\mathrm{CH}_{2}$ groups between the pyrene and the carboxilic acid groups ( $n=0 \leftrightarrow 1$-pyrenecarboxylic acid (PAc), $n=1 \leftrightarrow 1$-pyreneacetic acid (PMeAc) and $n=3 \leftrightarrow 1$ pyrenebutyric acid (PPrAc) (Figure ${ }^{\wedge \wedge} 1<x$ figr $1>c$ and Figure $\left.{ }^{\wedge \wedge} S 3\right)$. This was achieved following to protocol 1 , by dipping MWCNT films $\left(10^{\wedge \wedge} \mathrm{mg}\right)$ in an ethanol solution as described (see experimental section). MWCNT-PAc has the highest adsorption loading $\left(63 \times 10^{<\mathrm{M}->12 \wedge \wedge} \mathrm{mol} / \mathrm{cm}^{2}\right)$, that steadily drops as $n$ increases from $n=0$ to 3 (PPrAc), reaching a value close to that of the pyrene (Pyr, $45 \times 10^{<\mathrm{M}->12 \wedge \wedge} \mathrm{mol} / \mathrm{cm}^{2}$ ). Interestingly, replacing PMeAc 
by its lithiated salt PMeALi strongly decreases the molecular loading from $56 \times 10^{<\mathrm{M}->12}$ to $41 \times 10^{<\mathrm{M}->12 \wedge \wedge} \mathrm{mol} / \mathrm{cm}^{2}\left(\right.$ Figure $\left.^{\wedge \wedge} 1<\mathrm{xfigr} 1>\mathrm{c}\right)$.

To obtain more insight into the interaction we explore these systems with density functional theory. We can crudely estimate the experimental molecular surface coverage using the experimental pyrene surface concentration of $45 \times 10^{<\mathrm{M}->12 \wedge \wedge} \mathrm{mol} / \mathrm{cm}^{2}$, and our estimated pyrene molecule surface area (see method). This implies $\sim 0.18$ pyrene layers if parallel stacked to the surface, and $\sim 0.07 / 0.10$ layers for lateral/vertical stacking (Figure ${ }^{\wedge} \mathrm{S} 1$ ). Given the low molecular surface concentration, we modelled the adsorption of isolated molecules on graphene in a parallel configuration, as a first approximation to the system of study.

The molecules all bind strongly to graphene lying flat, $\pi$-stacking with the graphene surface with slight preference for $\mathrm{AB}$-stacking configuration, presenting the functional group extended and parallel to the surface in their most stable configurations (Figure ${ }^{\wedge \wedge} 2<$ figr $2>$ ). A complete comparison of the binding energies with respect to the different stacking configurations explored can be found in the supporting information (Figure ${ }^{\wedge} \mathrm{S} 4$ and Table ${ }^{\wedge \wedge} \mathrm{S} 1$ ). Where available our binding energies are in good agreement with previous literature values. ${ }^{[30]}$

The binding strength increases with molecule size as expected (Table^^ $1<\operatorname{tabr} 1>)$. The low-density energies calculated here represent minimum binding energies which could be higher. Despite the low surface molecular concentrations in the prepared electrodes, intermolecular forces can produce agglomerates leading to a non-homogeneous distribution of molecules on the electrode. Furthermore, the actual local concentration at the surface of the electrode film may be higher if the molecules are not able to efficiently penetrate into the inner part of the electrode film. In such scenarios, intermolecular interactions and packing considerations should be taken into account. Unfunctionalized pyrene can be packed in other 
configurations such as staggered or herringbone-type. ${ }^{[31]}$ Polar interactions may also arise between the substituted pyrene derivatives, and hydrogen bonds can form between the carboxylic groups of neighboring molecules. Competition between $\pi$ - $\pi$ stacking and hydrogen bonding influences the packing order of pyrene derivatives, that can undergo intermolecular hydrogen bonding on graphene. ${ }^{[32]}$ These molecules tend to aggregate more than nonhydrogen bonding derivatives, for example PAc molecules can form a herringbone structure of hydrogen bonded bilayers. Large functional groups may also mitigate molecules stacking on top. ${ }^{[33]}$

In our calculations, as expected for a low surface coverage, the molecules lie flat on the graphene sheet with the substituent lying flat in an extended conformation. In this case the functional group interacts with the graphene sheet, explaining the correlation of binding energy with molecular size. However, the $\langle\mathrm{C}-\rangle\left(\mathrm{CH}_{2}\right)_{n} \mathrm{COOH}$ substituents could present different non-interacting conformations, which might be favored under experimental conditions due to conformational entropy. Additionally, at high local surface concentration on the electrode film, such conformations could allow more compact molecular packing. In this case the higher number of interacting molecules would compensate for a lower individual molecular binding energy associated with the loss of substituent/MWCNT interaction, leading to a net gain in binding energy per unit of surface. In order to partially take into account this scenario, we have calculated binding energies for alternative PMeAc and PPrAc conformers with the substituents pointing upward (Table $\mathrm{e}^{\wedge \wedge} 1<\mathrm{xtabr} 1>$ ), effectively removing the contribution of the substituent to the binding energy (Figure ${ }^{\wedge} \mathrm{S} 5$, Table ${ }^{\wedge} \mathrm{S} 2$ and Table ${ }^{\wedge \wedge} \mathrm{S} 3$ ). In this case, only the first $<\mathrm{C}->\mathrm{CH}_{2}<\mathrm{C}$ - $>$ group of the alkyl chain, directly attached to the pyrene moiety, is in direct contact with the graphene surface. This conformational change increases the total energy by $\sim 0.14^{\wedge} \mathrm{eV}$ and $\sim 0.33--0.35^{\wedge} \mathrm{eV}$ for PMeAc and PPrAc respectively, with respect to the corresponding extended conformer. This energy change comes almost entirely from surface interaction since the energy differences between these 
different molecular conformers without graphene present are only $\sim 0.001^{\wedge \wedge} \mathrm{eV}$ and $\sim 0.03^{\wedge} \mathrm{eV}$ for PMeAc and PPrAc respectively. The binding energies for all molecules are now very similar, with pyrene presenting a lower absolute value.

We might expect modulation of the binding energy associated with the pyrene fragment due to the the electron-withdrawing/donation capability of the substituents. Unfortunately, these binding energies cannot be used for this purpose, since the different species have different numbers of atoms in contact with graphene. Only PMeAc and PPrAc share the same atoms in contact with graphene, and their similar binding energies suggest the change in functional group does not play a significant role. The lower binding energy observed for pyrene could just be due to the smaller number of atoms in contact with graphene in comparison with the rest of the species. Although these binding energies present a better correlation with the experimental loadings, the energy differences are very small to explain the experimental differences. Given the simplicity of these models, a complete explanation of the trends observed in the surface concentrations would need to take into account other factors such as intermolecular interactions and packing, as pointed out previously, solvent effects and thermal and entropic contributions. The drop of loading below that of pyrene upon replacement of PyrMeAc by its negatively charged $\mathrm{Li}^{+}$salt $\left(\right.$ PyrMeCOO $\left.<\mathrm{C}->\mathrm{Li}^{+}\right)$, which is expected to induce electrostatic repulsions, seems to support this point.

After adsorption of the molecules (Protocol 1 and Figure ${ }^{\wedge} 1<x$ figr $\left.1>c\right)$, MWCNTbased electrodes $\left(\mathrm{C}_{\mathrm{ad}} \mathrm{Pyr}, \mathrm{C}_{\mathrm{ad}} \mathrm{PrAc}, \mathrm{C}_{\mathrm{ad}} \mathrm{PMeAc}\right.$, and $\left.\mathrm{C}_{\mathrm{ad}} \mathrm{PPr} A \mathrm{c}\right)$ were electropolymerized in the LP-30 electrolyte at a current density of $1^{\wedge \wedge} \mathrm{A} / \mathrm{g}$ with a cut-off potential of $4.4^{\wedge \wedge} \mathrm{V} \mathrm{vs} . \mathrm{Li}^{+} / \mathrm{Li}$. Figure $^{\wedge} 3<<$ figr $3>$ a shows the first charge-discharge cycle, and Figure ${ }^{\wedge} 3<x f i g r 3>b$ the selected region where polymerization starts (first derivative plots, $\mathrm{d}\left(Q<\mathrm{C}->Q_{0}\right) / \mathrm{d} V$, are given Figure $\left.^{\wedge \wedge} \mathrm{S} 6 \mathrm{a}\right)$. In all cases the electrode potential increases sharply just after the open circuit 
potential, due to double layer charging of CNTs followed by oxidation of adsorbed molecules. Taking the pyrene oxidation potential as a reference $\left(4.14^{\wedge \wedge} \mathrm{V}\right)$, PAc lies $+0.13^{\wedge} \mathrm{V}$ vs. Pyrene at $4.27^{\wedge \wedge} \mathrm{V}$. The difference is much less pronounced for PMeAc $\left(+0.05^{\wedge \wedge} \mathrm{V}\right.$ vs. Pyrene at $\left.4.19^{\wedge \wedge} \mathrm{V}\right)$ and effectively negligable for $\operatorname{PPrAc}\left(+0.02^{\wedge \wedge} \mathrm{V}\right.$ vs. Pyrene, $\left.4.16^{\wedge \wedge} \mathrm{V}\right)$. Inductive and mesomeric effects of both the $\mathrm{COOH}$ (electron-withdrawing) and alkyl chain (electrondonor) tend to destabilize and stabilize respectively the pyrene radical cation, and so DFT calculations were conducted to rationalize the contribution of these effects. Figure ${ }^{\wedge} 4<$ figr $4>$ shows the calculated density of states and partial density of states as projected onto the graphene and molecule for each species bound to graphene, along with the energy levels of the equivalent isolated molecules. We can observe that the HOMO and LUMO levels of PAc are significantly lower than the corresponding levels of the rest of the molecules. In addition, the PDOS calculation shows that there is some contribution of the functional group fragment in both levels of PAc. These features are consistent with the combined inductive and resonance electron-withdrawing nature of the carboxylic acid substituent. ${ }^{[34,35]}$ Therefore, we should expect the oxidation of PAc to be energetically less favorable than for the rest of species. Finally, we observe that all HOMO levels are shifted to lower values when the molecules are on graphene, without affecting the original qualitative order between the HOMO levels of the different molecules. Further PDOS decomposition shows HOMO and LUMO states have $p_{\mathrm{z}}$ character. In addition, it can be observed that, in general, the pyrene states showing mainly $p_{z}$ character present a significant energy dispersion in the regions where the graphene PDOS present a dominant $p_{z}$ character (Figure ${ }^{\wedge} \mathrm{S} 8$ ). We attribute this to the interaction between the conjugated $p_{z}$ orbitals of pyrene and graphene. Given that two distinct $\mathrm{AB}$ stacking configurations exist for the substituted pyrene species (Figure ${ }^{\wedge} \mathrm{S} 4$ ), we finally tested the dependence of the electronic structure with respect to the stacking configuration by calculating the PDOS of PAc in its alternative AB configuration finding similar results (Figure ${ }^{\wedge \wedge} \mathrm{S} 7$, Figure ${ }^{\wedge \wedge} \mathrm{S} 8$ and Figure ${ }^{\wedge \wedge} \mathrm{S} 9$ ). Importantly, in all cases the Fermi 
level lies between the HOMO and LUMO of all monomers and no significant energy shifts are observed upon functionalization. This suggests that doping of graphene by the monomers, through a charge transfer mechanism, is not occurring. ${ }^{[36]}$ More in depth discussions regarding charge transfer mechanism as well as impact of the surface interaction between molecules and MWCNT on the pyrene redox potential are reported below Figure ${ }^{\wedge} \mathrm{S} 8$.

The negative HOMO energies can be interpreted as an estimation of the gas phase ionization energies (IEs). ${ }^{[37]}$ IEs estimated from DFT HOMOs using approximate xcfunctionals tend to underestimate experimental IEs and those computed with high-level theoretical methods, ${ }^{[37-41]}$ as reflected by comparison of the calculated IE value of pyrene in this work $\left(5.066^{\wedge \wedge} \mathrm{eV}\right)$ with its gas phase experimental vertical IE $\left(\sim 7.41^{\wedge \wedge} \mathrm{eV}\right) .{ }^{[42]}$ Despite the inaccuracy of this method, DFT HOMO energies are linearly correlated with experimental $\mathrm{IEs}^{[38]}$ and HOMO energies calculated with more accurate methods, ${ }^{[40,41]}$ Therefore, we can expect the relative order of IEs obtained from the HOMO values in this work to be correct. The trend observed in the calculated HOMO energies in Table^^ $1<x \operatorname{tabr} 1\rangle$ suggests that the $<\mathrm{C}->\mathrm{COOH}$ substituent behaves as a strong electron-withdrawing group, while $<\mathrm{C}$ $>\left(\mathrm{CH}_{2}\right)_{n} \mathrm{COOH}$ substituents behave as weak donor groups, since they lower and raise, respectively, the energy of the HOMO with respect to the parent pyrene molecule. The donor strength of $\langle\mathrm{C}-\rangle\left(\mathrm{CH}_{2}\right)_{n} \mathrm{COOH}$ substituents increases with $n$ due to the decreased influence of the terminal electron-withdrawing $<\mathrm{C}->\mathrm{COOH}$ group. This is in agreement with trends observed in experimental IEs of benzene and naphthalene derivatives bearing $\langle\mathrm{C}->\mathrm{COOH}$ and $<\mathrm{C}->\mathrm{CH}_{2} \mathrm{COOH}$ substituents, ${ }^{[43]}$ meta/para-orienting effects in the nitration of analogous benzene derivatives ${ }^{[44--47]}$ and available Hammett substituent constants. Previous studies have found linear correlations between experimental IEs of naphthalene and benzene derivatives and Hammett substituent constants, ${ }^{[43]}$ suggesting that these constants are transferable to polyaromatic systems. Available $\sigma_{\mathrm{p}}\left({\sigma_{\mathrm{p}}}^{+}\right)$Hammett substituent constants for $<\mathrm{C}->\mathrm{COOH}$, $+0.44,{ }^{[48]}+0.45(+0.42),{ }^{[49]}$ and for $\left\langle\mathrm{C}->\mathrm{CH}_{2} \mathrm{COOH},<\mathrm{M}->0.07^{[48]}(<\mathrm{M}->0.01)^{[49]}\right.$ are 
consistent with the interpretation of the electron-withdrawing/donor nature of the substituents obtained from the analysis of HOMO energies. Unfortunately, we did not find Hammett substituent constants for the remaining $\langle\mathrm{C}-\rangle\left(\mathrm{CH}_{2}\right)_{3} \mathrm{COOH}$ substituent, and the closest available one was for $\left\langle\mathrm{C}->\left(\mathrm{CH}_{2}\right)_{2} \mathrm{COOH}\right.$, for which a $\sigma_{\mathrm{p}}$ value of $\left\langle\mathrm{M}->0.07,{ }^{[48,49]}\right.$ equal to that of $\left\langle\mathrm{C}->\mathrm{CH}_{2} \mathrm{COOH}\right.$ was tabulated. An excellent linear correlation $\left(R^{2}>0.99\right)$ is found between IEs estimated from the HOMO values and the available $\sigma_{\mathrm{p}}$ and $\sigma_{\mathrm{p}}{ }^{+}$substituent constants for the molecules in both isolated and interacting with graphene models (Figure $\left.{ }^{\wedge} \mathrm{S} 10\right)$. The donor strength of $\langle\mathrm{C}-\rangle\left(\mathrm{CH}_{2}\right)_{n} \mathrm{COOH}$ substituents would approach that of an alkyl chain as $n$ becomes larger. Thus, we can take the value of the substituent constant of the ethyl group as a lower boundary for the actual value of the constant associated with the $<\mathrm{C}->\left(\mathrm{CH}_{2}\right)_{3} \mathrm{COOH}$ substituent. Indeed, assuming this limit value as the true value for the $<\mathrm{C}-$ $>\left(\mathrm{CH}_{2}\right)_{3} \mathrm{COOH}$ substituent maintains the excellent linear correlation with $\sigma_{\mathrm{p}}$ constants $\left(R^{2}>0.99\right)$, while a slightly weaker correlation with ${\sigma_{\mathrm{p}}}^{+}$constants is obtained $\left(R^{2}=0.94\right)$.

Calculation of oxidation potentials requires the calculation of oxidation free energies in solution, which are usually carried out by separating gas phase oxidation free energies and changes in free energies of solvation using a thermodynamic cycle, ${ }^{[40,50]}$ Computing gas phase free energies involves calculating the adiabatic IE at $0^{\wedge \wedge} \mathrm{K}$ including vibrational zero point energies (ZPE) and thermal and entropic contributions..$^{[40,50]}$ However, IEs and HOMO energies can be linearly correlated with experimental oxidation potentials for families of sufficiently similar species, ${ }^{[50--53]}$ Furthermore, a linear correlation has been also found for a small set of pyrene derivatives. ${ }^{[54]}$ Additionally, linear relationships have also been established between redox potentials and Hammett substituent constants, ${ }^{[55-57]}$ including, pyrene derivatives. ${ }^{[54,58]}$ Comparing the calculated HOMO energies (Table ${ }^{\wedge \wedge} 1<x$ tabr 1$\rangle$ ) with the experimental oxidation potentials shows we correctly capture the effect of the electronwithdrawing carboxylic substituent and its mitigated intensity with increasingly longer alkyl bridge. However, as observed for the binding energy values (Table ${ }^{\wedge \wedge} 1<x \operatorname{tabr} 1>$ ), the rank 
observed for the pyrene oxidation potential does not match that expected from calculated HOMO values which predict pyrene to be slightly more stable than PMeAc against oxidation $\left(\right.$ Table $\left.{ }^{\wedge} 1<x t a b r 1>\right)$. When excluding pyrene, measured oxidation potentials show a very good and satisfactory linear correlation with the calculated IEs (-HOMO) $\left(R_{\mathrm{IE}}{ }^{2}>0.99\right)$ for the molecules in both isolated and interacting with graphene models (Figure ${ }^{\wedge} \mathrm{S} 11 \mathrm{a}, \mathrm{b}$ ), as well as with Hammett substituent constants $\sigma_{\mathrm{p}}\left({\sigma_{\mathrm{p}}}^{+}\right)\left(R_{\text {Hammett }}{ }^{2}=0.98\right)$, assuming the substituent constant value of the ethyl group for the $\langle\mathrm{C}-\rangle\left(\mathrm{CH}_{2}\right)_{3} \mathrm{COOH}$ substituent (Figure ${ }^{\wedge} \mathrm{S} 10$ ). However, when including pyrene to the linear regressions, the $R^{2}$ values drop to $R_{\mathrm{IE}}{ }^{2}=0.86$ (0.88) with respect to the calculated IEs using the isolated molecules (graphene functionalized) model and to $R_{\text {Hammett }}^{2}=0.77(0.70)$ with respect to the $\sigma_{\mathrm{p}}\left(\sigma_{\mathrm{p}}{ }^{+}\right)$substituent constants. Considering only the species for which $\sigma_{\mathrm{p}}\left({\sigma_{\mathrm{p}}}^{+}\right)$substituent constants are available (PMeAc, Pyr and PAc) a similar low correlation is found with $R_{\text {Hammett }}^{2}=0.75(0.84)$. A visual inspection of the plots shows that the data points corresponding to pyrene are associated with an unexpectedly low oxidation potential. These discrepancies observed in the linear relationship studies suggest that pyrene is behaving differently to the species bearing a $<\mathrm{C}$ $>\mathrm{COOH}$ group. In order to understand this, we need to look at the limitations of the models used. We are assuming that thermal and solvent contributions to the oxidation free energies are either small or constant, what leads to a linear relationship between IEs and oxidation potentials. This assumption seems reasonable as the oxidation process mainly occurs at the same site (the pyrene moiety) for all species, without any bond breaking/formation process. Additionally, the fact that the calculated IEs correlate well with Hammett substituent constants (which are generally derived from experimental equilibrium and kinetic constants) supports this approximation. However, it may be possible that changes in solvent interactions during oxidation could be different for pyrene due to the absence of the $<\mathrm{C}->\mathrm{COOH}$ group. Although the same behavior is observed when comparing the oxidation potentials with the Hammett substituent constants, we have to take into account the limited amount of data 
available for this study and that substituent constants are derived for a different aromatic system in a different solvent. In addition, we are modelling individual molecules, and therefore neglecting the effect of intermolecular interactions. As discussed before when analyzing the binding energy results, the local molecular concentration could be higher than expected at the surface of the electrode film, and molecules could aggregate due to intermolecular forces. The nature of the functional group would have an important role in these intermolecular interactions between molecules and in determining how they aggregate. Molecular aggregation can have a significant effect in the IE. Clustering of pyrene molecules reduces its IE by $\sim 0.5^{\wedge \wedge} \mathrm{eV}$ when dimerizing and up to $\sim 0.7^{\wedge \wedge} \mathrm{eV}$ for clusters of five or seven molecules. ${ }^{[59]}$ These IE changes are larger than the whole energy range for all our calculated IE values $\left(\sim 0.4^{\wedge} \mathrm{eV}\right)$. Furthermore, excluding PAc leaves a much smaller energy range of only $\sim 0.1^{\wedge \wedge} \mathrm{eV}$ for the remaining species Pyr, PMeAc and PPrAc. Therefore, differences in the supramolecular arrangement of the different species have the potential to change the relative order of their corresponding IEs and oxidation potentials. For example, the unfunctionalized pyrene molecules could interact by $\pi$ - $\pi$ stacking with each other in a more effective way compared with the functionalized species for which the large substituents may hinder this stacking. In addition, the presence of the $<\mathrm{C}->\mathrm{COOH}$ group may dictate a molecular arrangement for which the distances between pyrene moieties could be increased. In such scenarios, the IE of pyrene may drop below the IE of PMeAc and PPrAc leading to the observed lower oxidation potential of pyrene.

To gain a better insight, fluorescence spectroscopy was performed for all pyrene derivative supernatant solutions that serve to adsorb molecules on MWCNT. In principle, the emission of pyrene is characterized by two cases: ${ }^{[60]}$ a monomeric state which is characterized by a set of vibronic bands ( $\pi \rightarrow \pi^{*}$ transitions) with well-defined peaks in the $370--450^{\wedge \wedge} \mathrm{nm}$ region and a possible excited state dimer or "excimer" associated with a broad band centered at around $460^{\wedge \wedge} \mathrm{nm}$. Excimer formation is favored by a rigid and planar molecular structure, 
such as anthracene ${ }^{[61]}$ and pyrene. ${ }^{[62]}$ However, although these structures can form aggregates through $\pi$-overlap, the latter can be suppressed by steric hindrance using highly twisted and bulky substituents. ${ }^{[63]}$ In fact, the monomer emission of pyrene means the distance between molecules is longer than $10^{\wedge \wedge} \AA$ while the distance in excimer state is shorter than $10^{\wedge \wedge} \AA .^{[64]}$ Herein, spectra recorded for supernatant solutions (Figure $\left.{ }^{\wedge} \mathrm{S} 12 \mathrm{a}\right)$ show five main monomeric vibronic bands with well-defined peaks at $\sim 375,379,385,395$, and $410^{\wedge \wedge} \mathrm{nm}$ as well as the excimer band (exc) at around $460^{\wedge \wedge} \mathrm{nm}$. The excimer intensity (relative to the monomer intensity $I_{1}$ at $\sim 375^{\wedge \wedge} \mathrm{nm}$ ) significantly decreases upon introduction of the substituent and is minimum for the bulkier one. We note that the intensity of the PPrCOOH excimer band decreases with dilution of the solution. This highlights that the electron transfer responsible for the occurrence of the excimer stems from intermolecular exchange, at the expense of intramolecular effects in the case of functionalized molecules (Figure $\left.{ }^{\wedge} \mathrm{S} 12 \mathrm{~b}\right)$. The observed effects therefore support that $\pi$-overlap molecular aggregation of the pyrene moiety (which is likely a more relevant factor for the shifts in IE and HOMOs than the H-bonds aggregate type is) would indeed be mitigated by introduction of the $\left(\mathrm{CH}_{2}\right)_{n}<\mathrm{C}->\mathrm{COOH}$ group. A related factor to take into account is the effect on the IE of the stacking of the molecules on the surfaces of the CNTs. The shifts in the HOMO energies observed when the molecules interact with the graphene model increase the expected IEs $\sim 0.2^{\wedge \wedge} \mathrm{eV}$, very similar to the error of the calculated IE of pyrene with respect to the regression line. To illustrate this, we show that a good linear correlation $\left(R^{2}=0.986\right)$ between calculated IEs and oxidation potentials can be obtained by taking the value of the free model for pyrene, and the values of the graphene functionalized models for the rest of species (Figure $\left.{ }^{\wedge} \mathrm{S} 11 \mathrm{c}\right)$. Given the high sensitivity of the IEs with both intermolecular and graphene surface interactions, and the good correlations between the calculated IEs and the Hammett substituent constants, we consider possible differences in the supramolecular arrangement of the molecules on the surface of the CNTs to likely be the main factor responsible for the discrepancies between the trends in measured 
oxidation potentials and calculated IEs. Nevertheless, other factors such as solvent interactions cannot be ruled out, and further studies would need to be done in order to better understand the unexpected results.

As pointed in Ref. [12] oxidative polymerization of pyrene occurs in two steps: firstly the monomer oxidation into radicals which concurrent proton release, and secondly the covalent attachment of the radicals into oligomers of different size and shape. The associated excess positive charge formed in the polymer is stabilized by delocalization within the resulting oligomers. Thus, electrons can be reversibly extracted without proton release according to the well known reversible/redox p-doping/de-doping process. Counter-anions $\left(\mathrm{PF}_{6}{ }^{<\mathrm{M}->}\right)$ from the electrolyte are reversibly inserted during this process to balance positive charge. In this way most of the initial charging cycle is associated with irreversible electrons consumption that is balanced by proton release, with the remainder, and all subsequent cycles involving electrons from reversible redox reaction/p-doping. The molar ratio of irreversible transferred electrons (protons) to adsorbed molecules is referred to as the "irreversible electron transfer" (IRET) in the following sections, while the ratio of reversible electrons $\left(\mathrm{PF}_{6}{ }^{<\mathrm{M}->}\right)$ to adsorbed molecules is referred to as the "reversible electron transfer" (RET). $<\mathrm{ffr} 1>$

$<\mathrm{ff} 1>p \mathrm{M} \rightarrow(<\mathrm{C}->\mathrm{M}<\mathrm{C}->)_{p}+p \cdot x \mathrm{H}^{+}+p \cdot x \mathrm{e}^{<\mathrm{M}->}<\mathrm{ZS}>(1)$

Reaction (1) illustrates the irreversible oxidative oligomer formation along with proton release that takes place during the initial cycle, where $\mathrm{M}$ is the monomer, $p$ is the number of moles of monomer, and $x$ the IRET, i.^e., the number of protons released per monomer. The value of $x$ depends on the molecular structure of the oligomer and is discussed later.

Reaction (1) is followed by reversible p-doping as indicated in Reaction (2): $<f f r 2>$ $<\mathrm{ff} 2>(<\mathrm{C}->\mathrm{M}<\mathrm{C}->)_{p}+p \cdot y \mathrm{PF}_{6}{ }^{<\mathrm{M}->} \leftrightarrow\left[(<\mathrm{C}->\mathrm{M}<\mathrm{C}->)_{p}{ }^{\mathrm{x}+}, p \cdot x \mathrm{PF}_{6}{ }^{<\mathrm{M}->}\right]+p \cdot y \mathrm{e}^{\langle\mathrm{M}->}<\mathrm{ZS}>(2)$ 
In this case $y$ is the RET, i.^e. the number of electrons released per repeating units $M$ in the adsorbed polymer $(<\mathrm{C}->\mathrm{M}<\mathrm{C}->)_{p}$. In order to obtain better insight into the electropolymerization process, quantification of IRET and RET is essential. RET is determined from the $50^{\text {th }}$ discharge capacity (Figure ${ }^{\wedge} \wedge 3<$ xfigr $3>c$ ), first derivative plots, $\mathrm{d}\left(Q^{<\mathrm{C}->} Q_{0}\right) / \mathrm{d} V$, are given Figure^^ $\left.\mathrm{S} 6 \mathrm{~b}\right)$ after subtracting the double layer capacity. IRET is determined from the capacity on $1^{\text {st }}$ charge after subtracting the RET. As reported in Table $\mathrm{A}^{\wedge} 2<$ tabr2 $>$, IRET increases in the order $\operatorname{Pyr}<\mathrm{C}->\mathrm{Ac}<\mathrm{C}->\mathrm{Li}<\mathrm{Pyr}<\mathrm{PAc}<\mathrm{PMeAc}<\operatorname{PPrAc}$. This trend is repeated in the size of a small hump in the first discharge cycle between 2.5-$3.0^{\wedge \wedge} \mathrm{V}$ vs. $\mathrm{Li}^{+} / \mathrm{Li}$, corresponding to a reduction of protons.

In the following we propose to use the IRET values to shed light on the polymerization process. Indeed, in the case of linear oligomers containing p pyrene moieties the IRET is expected to be $2(p--1) / p$, and hence for infinite linear type polymerization, IRET will tend to 2 . In Table $\mathrm{e}^{\wedge} 2<x$ tabr2 $>$ the observed IRET values are all greater than 2 and therefore, unlike proposed elsewhere ${ }^{[12]}$ cannot be explained here by linear polymerization. Instead, we believe ring fusion type polymerization as shown in Figure ${ }^{\wedge \wedge} 5<$ figr5> and Figure $^{\wedge} \mathrm{S} 13$ must be considered to explain the values of IRET. The observed IRET for Pyr (3.2) and PMeAc (2.9) indicate a tetramer and trimer structure respectively (Figure^^^S13) while the IRET of PAc (2.1) suggests a dimer. Interestingly, PPrAc shows the highest IRET of $4.4\left(\right.$ Table $^{\wedge \wedge} 2<x$ tabr2>. Considering this IRET values as average, several hypothetical oligomers of PPrAc can be proposed (Figure ${ }^{\wedge \wedge}$ S13d--h) with IRETs ranging from 7 to 4.4. Interestingly, two of them bring into play the first carbon as well as protons from the alkyl chain (Figure $\left.{ }^{\wedge} \mathrm{S} 13 \mathrm{~g}-\mathrm{-h}\right)$. The contribution of this specific alkyl carbon is justified by the differences in atomic Mulliken charges (see below). Therefore, it appears that the structure and molecular weight $\left(M_{\mathrm{w}}\right)$ of the oligomers depend on the attractive strength of the $\mathrm{COOH}$ substituent: the electron-withdrawing inductive effect of the $\mathrm{COOH}$ group pushes the onset potential towards the cut-off potential of the electrolyte which in-turn limits the extent of the 
polymerization reaction to low $M_{\mathrm{w}}$ oligomers and low IRET. Accordingly, decreasing the effect of the $\mathrm{COOH}$ by increasing the alkyl chain length results in higher Mw and IRET. Our results also indicate protons from the alkyl chain can also be removed (Figure ${ }^{\wedge \wedge} \mathrm{S} 13 \mathrm{~g}-\mathrm{h}$ ).

Further insight into possible polymerization mechanisms can be obtained from DFT calculations. The change in charge distribution upon extraction of an electron for each species, and the spin distribution of the resulting cation is shown in Figure $\wedge^{\wedge} 6<$ figr6>. In general, electron extraction remains largely localized in the pyrene moiety irrespective of the attached functional group. In the case of PAc, significant electronic charge is also extracted from the carboxylic carbon atom. To a lesser extent, some charge is extracted as well from the functional group in PMeAc and PPrAc, with the carboxylic group having a lesser participation in the subtracted charge as it is further from the pyrene moiety. This is in agreement with the participation of the pyrene moiety and functional group in the HOMO observed in the PDOS plots (Figure ${ }^{\wedge} 4<x$ figr $4>$ ). This suggests that oligomerization and polymerization will primarily involve the pyrene moiety. Additionally, when the cation is formed, the resulting spin is mostly localized in the pyrene moiety, in particular, on the carbon atoms at locations 1, 3, 6 and 8, suggesting these are likely attack sites for oligomerization.

While the actual mechanism of the polymerization process can be complex, we can obtain some clues from the presented Mulliken analysis. We could expect the mechanism to be a sequence of radical additions and proton removal reactions. During the first steps of the process, we would expect that the formation of new $\mathrm{C}<\mathrm{C}->\mathrm{C}$ bonds by radical reactions would involve participation of pyrene atoms with highest spin density (1, 3,6 and 8). On the other hand, removing electronic charge from a specific site would increase the acidity of this site, in comparison with the neutral monomer. Therefore, we may expect that sites with a higher positive $\Delta Q$ value would be more likely to be involved in an initial proton removal 
step. Although this mainly involves sites from the pyrene moiety, it is interesting to observe that some electron removal also occurs at the alkyl chain, especially at the alkyl $\mathrm{C}$ atom directly connected with the pyrene moiety in PPrAc. Thus, these alkyl sites also have the potential to participate in the polymerization process (Figure ${ }^{\wedge} \mathrm{S} 13 \mathrm{~g}-\mathrm{-h}$ ).

Turning now to the values of RET values (Table^^2<xtabr2>), it appears that the more extended the delocalized structure is (and therefore the higher the value of $n$ ), the higher the reversible capacity. This correlation is most likely associated with the stabilization of the radical by delocalization.

Interestingly, upon subtraction of the capacitive contribution of the MWCNT $\left(13^{\wedge \wedge} \mathrm{mAh} / \mathrm{g}\right)$, the reversible capacity calculated from the $50^{\text {th }}$ discharge cycle is $154^{\wedge \wedge} \mathrm{mAh} / \mathrm{g}$ for Pyr in $\mathrm{C}_{\mathrm{ad}} \mathrm{Pyr}$. In comparison, PMeAc in $\mathrm{C}_{\mathrm{ad}} \mathrm{PMe} A c$ and PPrAc in $\mathrm{C}_{\mathrm{ad}} \mathrm{PPrAc}$ show lower capacities i.^e., $133^{\wedge \wedge} \mathrm{mAh} / \mathrm{g}$ and $151^{\wedge \wedge} \mathrm{mAh} / \mathrm{g}$ respectively. The highest capacity observed for Pyr is due to its smaller Mw. However, it is noteworthy that the RET is much higher for PPrAc (1.6) and PMeAc (1.3) in comparison to Pyr (1.2) (details are reported in Table^^2<xtabr2>).

After adsorption and followed by "on-site" polymerization (Protocol 1, see experimental section), $\mathrm{C}_{\mathrm{ad}} \mathrm{PPrAc}$ demonstrates a capacity of $18^{\wedge \wedge} \mathrm{mAh} / \mathrm{g}$, corresponding to an improvement of nearly $40^{\wedge} \%$ (Table $\wedge^{\wedge} 2<x$ tabr $\left.2>\right)$ over untreated CNTs $\left(13^{\wedge \wedge} \mathrm{mAh} / \mathrm{g}\right)$, despite the presence of only $3--4^{\wedge \wedge} \mathrm{wt} \%$ of adsorbed molecules. On the other hand, $\mathrm{C}_{\mathrm{ad}} \mathrm{Pyr}$ and $\mathrm{C}_{\mathrm{ad}} \mathrm{PAc}$ show slightly lower capacities of $17^{\wedge \wedge} \mathrm{mAh} / \mathrm{g}$. The advantage of such low-levels of functionalization is they will not hamper the power characteristics of the resulting MWCNT/polymer composites as shown previously, ${ }^{[21]}$ however the capacities remain too low for practical applications. One strategy to enhance the functionalization yield is in-situ electropolymerization, by dissolving the monomers directly in the electrolyte of the battery, ${ }^{[21,22]}$ (Protocol 2, see experimental section). However, this requires many cycles, ${ }^{[21,22]}$ 
before the maximum capacity can be reached, as seen in Figure ${ }^{\wedge \wedge} \mathrm{S} 14$ for the PPrAc/MWCNT system which requires 200 cycles before doubling the initial capacity of the MWCNT electrode.

To overcome these issues we have developed a new protocol (Protocol 3) that consists of coating a suspension of MWCNT in NMP containing dissolved pyrene molecules directly onto an aluminum current collector (see Experimental Section for details). The resultant composite electrodes (CPyr, CPAc, CPMeAc, and CPPrAc) are then electrochemically polymerized at a constant current of $0.1^{\wedge \wedge} \mathrm{A} / \mathrm{g}$ in a potential window of $3--4.4^{\wedge \wedge} \mathrm{V} \mathrm{vs} . \mathrm{Li}^{+} / \mathrm{Li}$. Structural changes undegone by composite electrodes during electrochemical polymerization and cycling were characterized by scanning electron microscopy. Images of the initial CPMeAc electrode, and after the $1^{\text {st }}, 5^{\text {th }}$ and $100^{\text {th }}$ charge-discharge cycles are compared to that of the untreated MWCNT one in Figure ${ }^{\wedge \wedge} 7<$ figr $7>$. Before cycling, pyrene derivative are micro scale particles in the $1--5^{\wedge \wedge} \mu \mathrm{m}$ size mixed with MWCNT fibers (Figure ${ }^{\wedge \wedge} 7<x$ xigr $7>$ b-c). After the $1^{\text {st }}$ cycle, most of these grains have disappeared while deposits are observed on the walls of the MWCNTs in agreement the dissolution-reprecipitation steps expected during anodic oligomerization (Figure^^ $7<x$ figr $7>d$ ). From the $5^{\text {th }}$ cycle onward, this trend is pursued until conformal deposits coat MWCNT surface (Figure ${ }^{\wedge \wedge} 7<x$ xigr $\left.7>e--f\right)$.

Figure $^{\wedge \wedge} 8<$ figr $8>$ a shows the charge-discharge profile of different composite electrodes along with a blank electrode of untreated MWCNTs prepared using protocol 3 in a Li metal hybrid supercapacitor configuration. The highest capacity is $77^{\wedge \wedge} \mathrm{mAh} / \mathrm{g}_{\text {electrode }}$ $\left(116^{\wedge \wedge} \mathrm{F} / \mathrm{g}_{\text {electrode }}\right)$ for CPPrAc-55 (the last two digits of the sample name indicate the weight percentage of molecules in that composite), a nearly $500^{\wedge} \%$ increase over untreated MWCNTs. The capacity with Protocol 3 is twice that of the in-situ method (protocol 2) even in the initial cycles without any degradation in Coulombic efficiency (Figure $\left.{ }^{\wedge \wedge} \mathrm{S} 14\right)$. The capacity of the PPrAc molecules in CPPrAc-55 after removing the EDLC contribution is 
$128^{\wedge} \wedge \mathrm{mAh} / \mathrm{g}_{\mathrm{PPrAc}}$, lower than the $162^{\wedge \wedge} \mathrm{mAh} / \mathrm{g}_{\mathrm{PPrAc}}$ as obtained upon protocol $1\left(3.9^{\wedge} \mathrm{Wt} \%\right.$ of PPrAc). The difference is most likely due to lower electron transfer kinetics or anion/solvent diffusivity through the increased thickness of the deposit. This high capacity corresponds to a RET of 1.4 for CPPrAc-55, whereas CPMeAc-55 shows RET $=1.1$ and $Q=68^{\wedge} \mathrm{mAh} / \mathrm{g}_{\text {electrode }}$ $\left(102^{\wedge \wedge} \mathrm{F} / \mathrm{g}_{\text {electrode }}\right)$. A summary of capacity values and RET of all the molecules is given in Figure $^{\wedge \wedge} 8<$ xfigr $8>$ b.

The trends observed in capacity, IRET, and RET for Protocol 3 follow closely those of Protocol 1 as indicated in Table^^2<xtabr2>. However, the electrode capacities are much higher due to the larger $\mathrm{wt}^{\wedge} \%$ of pyrene molecules in the composite. As inferred from Figure $^{\wedge \wedge} 8<x$ xigr8 $>b$ and Table ${ }^{\wedge} \mathrm{S} 4$, CPPMeAc shows the best compromise in terms of capacity (approaching that of PPrAc), better power retention (Figure ${ }^{\wedge} \mathrm{S} 15$ ) and lower IRET (3.7 for CPPMeAc vs 5.2 for CPPrAc). It is worth mentioning that a compromise must be made in the electrode composition; while increasing functionalization increases faradic capacity, it also degrades the electrochemical kinetics (Figure ${ }^{\wedge} 8<x$ figr8 $>$ d). ${ }^{[65--69]}$ In order to find this optimal composition, the amount of 1-pyreneacetic acid (PMeAc) in the composites was varied from 15 to $95^{\wedge \wedge} \mathrm{wt} \%$ using Protocol-3. The corresponding charge-discharge profiles are shown and compared to pure PMeAc and MWCNTs in Figure ${ }^{\wedge} 8<<$ xfigr8 $>c$, with corresponding capacities are reported in Figure ${ }^{\wedge \wedge} 8<x$ figr $8>d$ (after subtraction of the MWCNT capacity for PMeAc-MWCNT composites). This shows that up to CPMeAc-28, PMeAc allows an excellent specific capacity of $137^{\wedge \wedge} \mathrm{mAh} / \mathrm{g}_{\text {molecule. }}$. The optimum capacity per mass of the whole electrode is reached for a molecule loading in the range of 55 to $70^{\wedge \wedge} \mathrm{Wt} \%$ at $0.1^{\wedge \wedge} \mathrm{A} / \mathrm{g}$ illustrating that higher loadings tend to mitigate the utilization of the molecule (and the power capability). At similar current load and total electrode loading, such electrode specific capacities fall in the range of that associated with a poly $(2,2,6,6-$ tetramethyl-1-piperinidyloxy-4-yl methacrylate), referred to as PTMA, and (SWCNTactivated carbon) composite. ${ }^{[70]}$ Interestingly, however, if the average potential on discharge 
is in the present case approx. $500^{\wedge \wedge} \mathrm{mV}$ lower than that arising from the use of PTMA, ${ }^{[70]}$ the higher specific capacity associated with pyrene derivatives allows a significantly larger gain of specific capacity per mass of electrode $\left(+320^{\wedge} \%\right.$ herein from extrapolation of Figure ${ }^{\wedge} 3<x$ figr $3>$ d, vs. $+65^{\wedge} \%$ in Ref. [70], both at $40^{\wedge \wedge}$ wt $\%$ molecule loading) therefore indicating even better performance could arise from optimization of the carbon substrate capacitance. Interestingly, a PMeAc-70 electrode still maintains a capaciy of $50^{\wedge \wedge} \mathrm{mAh} / \mathrm{g}_{\text {electrode }}\left(75^{\wedge \wedge} \mathrm{F} / \mathrm{g}_{\text {electrode }}\right)$ at a $20^{\wedge \wedge}$ times higher current load $\left(2^{\wedge \wedge} \mathrm{A} / \mathrm{g}_{\text {electrode }}\right)$ (Figure $\left.{ }^{\wedge} \mathrm{S} 15\right)$ which is nearly $4^{\wedge \wedge}$ times more than the bare MWCNT electrode, and $1.7^{\wedge}$ times more than a PPAAc-70 electrode of same specific loading. It should be noticed that n-type electroactive molecules paired with Li counter-ions such as calix[4]arene have also been proposed to functionalize either $\mathrm{SiO}_{2}$ or carbon susbtrate. ${ }^{[71]}$ In this case, although functionalization allowed to mitigate the molecule solubility the electrode specific capacity was restricted to nearly $40^{\wedge \wedge} \mathrm{mAh} / \mathrm{g}\left(0.096^{\wedge \wedge} \mathrm{A} / \mathrm{g}\right)$ with a lower average potential (nearly

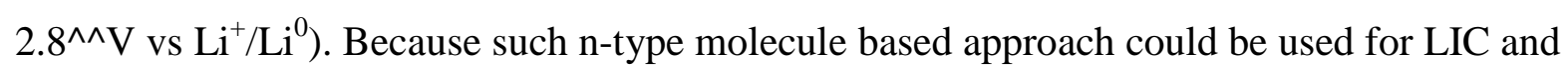
hybrid capacitors but also combined to Li or Na-ion battery electrode to compensate for SEI formation, ${ }^{[72,23]}$ it could be re-explored in the near future using recently discovered Li salts that show performance comparable to that of $\mathrm{LiFePO}_{4}$ both in terms of specific capacity and average potential. ${ }^{[73,74]}$

\section{Conclusions}

The effect of functional groups on pyrene adsorption and associated electropolymerization mechanisms on MWCNT electrodes, as well as resulting electrochemical charge-storage properties in a Li metal hybrid supercapacitor configuration are reported. Both the alkyl chain length of pyrene functional groups and the composite electrode formulation protocol are considered. Self-adsorption of pyrene derivatives on MWCNT from supernatant solution results in loadings in the range of a few $10^{<\mathrm{M} \text { - }}$ 
$>11 \wedge \wedge \mathrm{mol} / \mathrm{cm}^{2}$. The latter steadily drops as the length of the alkyl chain increases reaching a value close to that of pyrene. Calculations show that all pyrene moieties show strong surface binding to graphene adopting preferentially $\mathrm{AB}$ stacking. Binding is most energetically favorable with extended functional groups interacting with graphene, the binding energy increasing with functional group size. When the functional groups are not in surface contact, the conformers have similar binding energies to each other. Although calculated binding energies present a fair correlation with the experimental loadings, the energy differences are very small to explain the experimental differences.

Electronic structure calculations show that in every case the Fermi energy lies between the molecular HOMO and LUMO indicating no charge transfer in the ground state. HOMO values suggests the $<\mathrm{C}$ - $>\mathrm{COOH}$ group is strongly electron-withdrawing while the $<\mathrm{C}$ $>\left(\mathrm{CH}_{2}\right)_{n} \mathrm{COOH}$ are slightly electron-donating. We find good linear correlation between predicted IEs (-HOMO) and Hammett substituent constants. However, correlations between measured oxidation potentials and calculated IEs and Hammett substituent constants fail for pyrene. This could be explained by the sensitivity of the IE with intermolecular and surface interactions, the former being supported by fluorescence spectroscopy. Other factors not taken into account in the study such as solvent effects and thermal and entropic contributions to oxidation free energies should be also studied.

Calculated Mulliken charges shows that oxidation mainly affects the pyrene fragment, with significant electronic charge also extracted from the carboxylic carbon atom in the case of PAc. A smaller amount of charge is extracted as well from atoms in the functional group in PMeAc and PPrAc. Within the alkylic $\mathrm{C}$ atoms, the one directly connected to the pyrene moiety is the most affected. A higher radical character can be found in 1,3,6,8 sites. These sites are the most likely to participate in possible initial radical reactions of the polymerization 
process, while sites that have been charged positively would be expected to participate in possible initial proton removal reactions.

Based on these calculation results, and accounting for the experimental values of the coulometric irreversibility, fusion rather than linear-type oligomerization mechanisms are described for the first time for each pyrene derivative. These findings directly allow rationalisation of the storage performance of these carbon composite electrodes since the longer alkyl chains lead to more extended functionzalized oligomers, and hence enhance pdoping (reversible capacity). In a Li metal hybrid supercapacitor configuration, best capacitypower compromise was found with $55^{\wedge \wedge} \mathrm{wt} \%$ Pyrene- $\left(\mathrm{CH}_{2}\right) \mathrm{COOH}$, resulting in a 5-fold capacity increase from $13^{\wedge \wedge} \mathrm{mAh} / \mathrm{g}_{\text {electrode }}\left(19^{\wedge \wedge} \mathrm{F} / \mathrm{g}_{\text {electrode }}\right)$ for untreated MWCNT to $68^{\wedge \wedge} \mathrm{mAh} / \mathrm{g}_{\text {electrode }}\left(102^{\wedge \wedge} \mathrm{F} / \mathrm{g}_{\text {electrode }}\right)$ between $2--4.4^{\wedge} \wedge \mathrm{V}$ vs. $\mathrm{Li}^{+} / \mathrm{Li}$.

\section{Supporting Information}

Additional references cited with the Supporting Information. ${ }^{\text {[75--87] }}$

\section{Acknowledgements}

B.A., T.B., and J.G. acknowledge the financial assistance provided by the $\langle$ cgs $>$ Agence Nationale de la Recherche $</$ cgs $>$ (ANR) Project No. $<$ cgn $>13-$ PRGE-0011</cgn $>$ and the $<$ cgs $>$ French Research Network on Electrochemical Energy Storage $</$ cgs $>$ (RS2E). C.P.E. and Y.S.B. have received funding from the <cgs> European Union's Horizon 2020 Research and Innovation program $</$ cgs $>$ under the Marie Sklodowska-Curie grant agreement No $<$ cgn $>642742</$ cgn $>$, and acknowledge the CCIPL (Centre de Calcul Intensif Pays de la Loire) where many of the DFT calculations were performed. J.G. is thankful to F. Odobel (CEISAM) for fruitful discussions.

\section{Conflict of Interest}

The authors declare no conflict of interest.

<lit1><jnl>M.^^R. Palacín, Chem. Soc. Rev. 2009, 38, 2565</jnl>. 
$<$ lit2><jnl>W. Luo, F. Shen, C. Bommier, H. Zhu, X. Ji, L. Hu, Acc. Chem. Res. 2016, 49, $231--240</ j n l>$.

$<$ lit3><book>B.^^E. Conway, Electrochemical Capacitors: Scientific Fundamentals and Technological Applications, Springer, 1999</book>.

$<$ lit4><jnl>V. Aravindan, J. Gnanaraj, Y.-S. Lee, S. Madhavi, Chem. Rev. 2014, 114, 11619-$11635</$ jnl $>$.

$<$ lit5><jnl>V. Aravindan, Y.^^S. Lee, S. Madhavi, Adv. Energy Mater. 2017, 7, $1602607</$ jnl $>$.

<lit6><jnl>M.^^S. Whittingham, Chem. Rev. 2004, 104, 10, 4271--4302</jnl>.

$<$ lit7><jnl>C. Liu, Z.^^G. Neale, G. Cao, Mater. Today 2016, 19, 109--123</jnl>.

$<$ lit8><jnl>E. Frackowiak, F. Béguin, Carbon 2001, 39, 937--950</jnl>.

<lit9><jnl>M. Sevilla, R. Mokaya, Energy Environ. Sci. 2014, 7, 1250--1280</jnl>.

$<$ lit10><jnl >L. Dong, W. Yang, W. Yang, Y. Li, W. Wu, G. Wang, J. Mater. Chem. A 2019, 7, 13810--13832</jnl>.

$<$ lit11 ><jnl>P. Simon, Y. Gogotsi, B. Dunn, Science 2014, 343, 1210--1211</jnl>.

$<$ lit12><jnl>J. ${ }^{\wedge}$ C. Bachman, R. Kavian, D. ${ }^{\wedge} J$. Graham, D. ${ }^{\wedge} Y$. Kim, S. Noda, D. ${ }^{\wedge} G$.

Nocera, Y. Shao-Horn, S.^^W. Lee, Nat. Commun. 2015, 6, 7040</jnl>.

$<$ lit13 $><$ jnl $>$ B. Anothumakkool, D. Guyomard, J. Gaubicher, L. Madec, Nano Res. 2017, 10, 4175--4200</jnl>.

$<$ lit14><jnl>A. Borenstein, S. Hershkovitz, A. Oz, S. Luski, Y. Tsur, D. Aurbach, J. Phys.

Chem. C 2015, 119, 12165--12173</jnl>.

$<$ lit15><jnl >N. An, F. Zhang, Z. Hu, Z. Li, L. Li, Y. Yang, B. Guo, Z. Lei, RSC Adv. 2015, 5, 23942--23951</jnl >. 
$<$ lit16 > <jnl >C. Ramirez-Castro, O. Crosnier, L. Athouel, R. Retoux, D. Belanger, T. Brousse, J. Electrochem. Soc. 2015, 162, A5179--A5184</jnl>.

$<$ lit17><jnl>C.^^A. Hunter, J.^^K.^^M. Sanders, J. Am. Chem. Soc. 1990, 112, 5525-$5534</$ jnl $>$.

$<$ lit18><jnl>R.^^J. Waltman, J. Bargon, Can. J. Chem. 1986, 64, 76--95</jnl>. $<$ lit19><jnl>R.^^J. Waltman, A.^^F. Diaz, J. Bargon, J. Phys. Chem. 1984, 88, 4343-$4346</$ jnl $>$.

$<$ lit20><jnl>R.^^J. Waltman, J. Bargon, J. Electroanal. Chem. 1985, 194, 49--62</jnl>. $<$ lit21 ><jnl>B. Anothumakkool, P.-L. Taberna, B. Daffos, P. Simon, Y. Sayed-AhmadBaraza, C. Ewels, T. Brousse, J. Gaubicher, J. Mater. Chem. A 2017, 5, 1488-$1494</$ jnl $>$.

$<$ lit22 ><jnl >L. Madec, A. Bouvrée, P. Blanchard, C. Cougnon, T. Brousse, B. Lestriez, D. Guyomard, J. Gaubicher, Energy Environ. Sci. 2012, 5, 5379--5386</jnl>. $<$ lit23 ><jnl>B. Anothumakkool, S. Wiemers- Meyer, D. Guyomard, M. Winter, T. Brousse, J. Gaubicher, Adv. Energy Mater. 2019, 9, 1900078</jnl>. $<$ lit24><jnl>M.^^J. Rayson, P.^^R. Briddon, Phys. Rev. B 2009, 80, 205104</jnl>. $<$ lit25><jnl>P.^^R. Briddon, M.^^^J. Rayson, Phys. Status Solidi B 2011, 248, 1309-$1318</$ jnl $>$. <lit26><jnl>P.^^R. Briddon, R. Jones, Phys. Status Solidi B 2000, 217, 131--171</jnl>. $<$ lit27><jnl>W.^^S. Su, T.^^C. Leung, C.^^T. Chan, Phys. Rev. B 2007, 76, 235413</jnl>. $<$ lit28><jnl>C. Hartwigsen, S. Goedecker, J. Hutter, Phys. Rev. B 1998, 58, 3641-$3662</$ jnl $>$. <lit29><jnl>H.^^J. Monkhorst, J.^^D. Pack, Phys. Rev. B 1976, 13, 5188--5192</jnl>. 
$<$ lit30><jnl $>$ S. Bailey, D. Visontai, C. ${ }^{\wedge}$ J. Lambert, M.^^R. Bryce, H. Frampton, D.

Chappell, J. Chem. Phys. 2014, 140, 054708</jnl>.

$<$ lit31 ><jnl>A. Camerman, J. Trotter, Acta Crystallogr. 1965, 18, 636--643</jnl >.

$<$ lit32><jnl>Y. Liu, L. Yuan, M. Yang, Y. Zheng, L. Li, L. Gao, N. Nerngchamnong, C. ${ }^{\wedge}$ T.

Nai, C. $.^{\wedge} S .{ }^{\wedge}$ S. Sangeeth, Y.^^P. Feng, C.^^A. Nijhuis, K.^^P. Loh, Nat. Commun.

$\mathbf{2 0 1 4}, 5,5461</ \mathrm{jnl}>$.

$<$ lit33><jnl>A.^^T. Haedler, H. Misslitz, C. Buehlmeyer, R.^^Q. Albuquerque, A. Köhler,

H.-W. Schmidt, ChemPhysChem 2013, 14, 1818--1829</jnl>.

$<$ lit34><jnl >M. Ottonelli, M. Piccardo, D. Duce, S. Thea, G. Dellepiane, J. Phys. Chem. A

2012, 116, 611--630</jnl >

$<$ lit35 $><$ jnl $>$ A. Kathiravan, M. Panneerselvam, K. Sundaravel, N. Pavithra, V. Srinivasan, S.

Anandan, M. Jaccob, Phys. Chem. Chem. Phys. 2016, 18, 13332--13345</jnl>.

$<$ lit36><jnl >H. Pinto, R. Jones, J.^^P. Goss, P.^^R. Briddon, Phys. Status Solidi A 2010, 207, 2131--2136</jnl >

$<$ lit37><jnl>E.^^J. Baerends, O.^^V. Gritsenko, R. van^^Meer, Phys. Chem. Chem. Phys. 2013, 15, 16408--16425</jnl >.

$<$ lit38><jnl>G. Zhang, C.^^B. Musgrave, J. Phys. Chem. A 2007, 111, 1554--1561</jnl>. $<$ lit39><jnl>H. Jiang, Y.-C. Shen, J. Chem. Phys. 2013, 139, 164114</jnl>.

$<$ lit40 $><$ jnl $>$ M. Isegawa, F. Neese, D.^^A. Pantazis, J. Chem. Theory Comput. 2016, 12, $2272--2284</$ jnl $>$.

$<$ lit41 ><jnl >R. Rodrigues^^Pela, A. Gulans, C. Draxl, J. Chem. Theory Comput. 2018, 14, 4678--4686</jnl>. 
<lit42><other> "NIST Chemistry WebBook," can be found under https://doi.org/10.18434/T4D303, n.d</other>.

$<$ lit43><jnl>L. Klasinc, B. Kovac, H. Gusten, Pure Appl. Chem. 1983, 55, 289--298</jnl>. $<$ lit44><jnl>P. Strazzolini, G. Verardo, F. Gorassini, A.^^G. Giumanini, Bull. Chem. Soc. Jpn. 1995, 68, 1155--1161</jnl >.

$<$ lit45><jnl>P. Strazzolini, A.^^G. Giumanini, A. Runcio, M. Scuccato, J. Org. Chem. 1998, 63, 952--958</jnl $>$.

$<$ lit46><jnl>A.^^R. Hajipour, A.^^E. Ruoho, Phosphorus Sulfur Silicon Relat. Elem. 2004, $179,221--226</ \mathrm{jnl}>$.

$<$ lit47><jnl>D.^^L. Yabroff, C. ${ }^{\wedge \wedge}$ W. Porter, J. Am. Chem. Soc. 1932, 54, 1199--1204</jnl>. $<$ lit48><book>D.^^D. Perrin, B. Dempsey, E. ${ }^{\wedge}$ P. Serjeant, in PKa Prediction for Organic Acids and Bases (Eds.: D.^^D. Perrin, B. Dempsey, E.^^P. Serjeant), Springer

Netherlands, Dordrecht, 1981, pp. 44--52</book>.

$<$ lit49><jnl>C. Hansch, A. Leo, R. ${ }^{\wedge}$ W. Taft, Chem. Rev. 1991, 91, 165--195</jnl>. $<$ lit50><book> J. Ho, M.^^L. Coote, C.^^J. Cramer, D. ${ }^{\wedge \wedge}$ G. Truhlar, in Organic

Electrochemistry: Revised and Expanded (Eds.: O. Hammerich, B. Speiser), CRC Press, Boca Raton, FL, USA, 2015, pp. 229--259.</book>.

$<$ lit51><book> C. Amatore, in Organic Electrochemistry (Eds.: O. Hammerich, B. Speiser), CRC Press, Boca Raton, FL, USA, 2015, pp. 3--96</book>. $<$ lit52><jnl >D.^^D. Méndez-Hernández, P. Tarakeshwar, D. Gust, T.^^A. Moore, A. ${ }^{\wedge}$ L. Moore, V. Mujica, J. Mol. Model. 2013, 19, 2845--2848</jnl>. $<$ lit53><jnl>L. Cheng, R.^^S. Assary, X. Qu, A. Jain, S.^^P. Ong, N.^^N. Rajput, K. Persson, L.^^A. Curtiss, J. Phys. Chem. Lett. 2015, 6, 283--291</jnl>. 
$<$ lit54><jnl>J.^^C. Bachman, R. Kavian, D.^^J. Graham, D.^^Y. Kim, S. Noda, D. ${ }^{\wedge}$ G.

Nocera, Y. Shao-Horn, S.^^W. Lee, Nat. Commun. 2015, 6, 7040</jnl>.

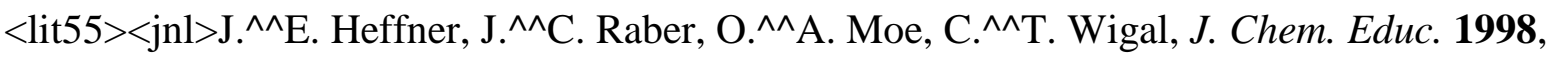
$75,365</$ jnl $>$.

$<$ lit56><book> O. Hammerich, B. Speiser, Eds., Organic Electrochemistry : Revised and Expanded, CRC Press, Boca Raton, FL, USA, 2015</book>.

$<$ lit57><jnl>L. Engman, J. Persson, C. ${ }^{\wedge}$ M. Andersson, M. Berglund, J. Chem. Soc. Perkin Trans. 2 1992, 1309--1313</jnl $>$.

$<$ lit58><jnl $>$ R.^^J. Waltman, J. Bargon, J. Electroanal. Chem. Interfacial Electrochem. 1985, 194, 49--62</jnl>.

$<$ lit59><jnl>C. Joblin, L. Dontot, G.^^A. Garcia, F. Spiegelman, M. Rapacioli, L. Nahon, P.

Parneix, T. Pino, P. Bréchignac, J. Phys. Chem. Lett. 2017, 8, 3697--3702</jnl>.

$<$ lit60 $><j n l>G . \wedge \wedge$ K. Bains, S.^^H. Kim, E. ${ }^{\wedge} J$. Sorin, V. Narayanaswami, Biochemistry 2012, 51, 6207--6219</jnl>.

$<$ lit61 $><$ jnl $>$ P.^^P. Neelakandan, D. Ramaiah, Angew. Chem. Int. Ed. 2008, 47, 8407-$8411</$ jnl $>$.

$<$ lit62><jnl>D. Mansell, N. Rattray, L.^^L. Etchells, C. ${ }^{\wedge \wedge}$ H. Schwalbe, A.^^J. Blake, E. ${ }^{\wedge}$ V.

Bichenkova, R.^^A. Bryce, C.^^J. Barker, A. Díaz, C. Kremer, S. Freeman, Chem.

Commun. 2008, 5161--5163</jnl $>$.

$<$ lit63><jnl>P.-I. Shih, C.-Y. Chuang, C.-H. Chien, E.^^W.-G. Diau, C.-F. Shu, Adv. Funct.

Mater. 2007, 17, 3141--3146</jnl >.

$<$ lit64><book>S.^^S. Lehrer, in Proteins: Structure, Function, and Engineering (Eds.: B. ${ }^{\wedge}$ B.

Biswas, S. Roy), Springer US, Boston, MA, 1995, pp. 115--132</book>. 
$<$ lit65><jnl>H. Lin, L. Li, J. Ren, Z. Cai, L. Qiu, Z. Yang, H. Peng, Sci. Rep. 2013, 3, $1353</$ jnl $>$.

$<$ lit66><jnl>E. Frackowiak, V. Khomenko, K. Jurewicz, K. Lota, F. Béguin, J. Power Sources 2006, 153, 413--418</jnl >

$<$ lit67 $><$ jnl $>$ B. Anothumakkool, A. ${ }^{\wedge \wedge}$ T..$^{\wedge}$ A. Torris, S. ${ }^{\wedge}$ N. Bhange, S. ${ }^{\wedge} M$ M. Unni, M. ${ }^{\wedge}$ V.

Badiger, S. Kurungot, ACS Appl. Mater. Interfaces 2013, 5, 13397--13404</jnl>. $<$ lit68 $><$ jnl $>$ B. Anothumakkool, A. ${ }^{\wedge \wedge}$ T..$^{\wedge}$ A. Torris, S.^^N. Bhange, M.^^V. Badiger, S. Kurungot, Nanoscale 2014, 6, 5944--52</jnl>.

$<$ lit69><jnl >V. Khomenko, E. Frackowiak, F. Béguin, Electrochim. Acta 2005, 50, 2499-$2506</$ jnl $>$.

$<$ lit70><jnl>A. Vlad, N. Singh, S. Melinte, J.-F. Gohy, P.^^M. Ajayan, Sci. Rep., 2016, 6, 22194--22199</jnl>.

$<$ lit71><jnl>B. Genorio, K. Pirnat, R. Cerc-Korosec, R. Dominko, M. Gaberscek, Angew.

Chem. Int. Ed. 2010, 49, 7222--7224</jnl>.

$<$ lit72><jnl>F. Holtstiege, P. Bärmann, R. Nölle, M. Winter, T. Placke, Batteries, 2018, 4, 4-$42</$ jnl $>$.

$<$ lit73><jnl>A. Jouhara, E. Quarez, F. Dolhem, M. Armand, N. Dupré, P. Poizot, Angew.

Chem. Int. Ed. 2019, 58, 15680--15684</jnl>.

$<$ lit74><jnl >J. Wang, A.^^E. Lakraychi, X. Liu, L. Sieuw, C. Morari, P. Poizot, A. Vlad, Nat. Mater. 2020, https://doi.org/10.1038/s41563-020-00869-1</jnl>.

$<$ lit75><jnl>A. Bondi, J. Phys. Chem. 1964, 68, 441--451</jnl>. 
$<$ lit76><book>P.^^G. Collins, P. Avouris, in Carbon Nanotubes: Quantum Cylinders of Graphene (Eds.: S. Saito, A. Zettl), Elsevier, Amsterdam-Oxford, 2008, pp. 49-$81</$ book $>$.

$<$ lit77><jnl>S. Arai, T. Kanazawa, Surf. Coat. Technol. 2014, 254, 224--229</jnl>.

$<$ lit78><jnl >B. Gong, A. Ikematsu, K. Waki, Carbon 2017, 114, 526--532</jnl>.

$<$ lit79><jnl>S. Naghdi, G. Sanchez-Arriaga, K. ${ }^{\wedge}$ Y. Rhee, J. Alloys Compd. 2019, 805, 1117$-1134</ \mathrm{jnl}>$.

$<$ lit80><jnl>W. Mu, G. Zhang, Z. Ou-Yang, Jpn. J. Appl. Phys. 2012, 51, 065101</jnl>. $<$ lit81 $><$ jnl $>$ K. Jurkiewicz, M. Pawlyta, A. Burian, $C$ 2018, 4, 68</jnl $>$.

$<$ lit82><jnl>S. Shallcross, S. Sharma, E. Kandelaki, O.^^A. Pankratov, Phys. Rev. B 2010, $81,165105</$ jnl $>$.

$<$ lit83 $><$ jnl $>$ S. Okuda, M. Shimatani, S. Fukushima, S. Ogawa, T. Ikuta, K. Maehashi, Proc. SPIE 2020, 11407, 114071^^ $\mathrm{J}</ \mathrm{jnl}>$.

$<$ lit84 $><$ jnl $>$ D. Weckbecker, S. Shallcross, M. Fleischmann, N. Ray, S. Sharma, O.

Pankratov, Phys. Rev. B 2016, 93, 035452</jnl>.

$<$ lit85><jnl >M. Sprinkle, D. Siegel, Y. Hu, J. Hicks, A. Tejeda, A. Taleb-Ibrahimi, P.

Le ${ }^{\wedge}$ Fèvre, F. Bertran, S. Vizzini, H. Enriquez, S. Chiang, P. Soukiassian, C. Berger, W. ${ }^{\wedge}$ A. de $^{\wedge \wedge}$ Heer, A. Lanzara, E. ${ }^{\wedge} H$. Conrad, Phys. Rev. Lett. 2009, 103, $226803</$ jnl $>$.

$<$ lit86><jnl>A. Luican, G. Li, A. Reina, J. Kong, R. ${ }^{\wedge}$ R. Nair, K.^^S. Novoselov, A.^^K. Geim, E. ${ }^{\wedge} Y$. Andrei, Phys. Rev. Lett. 2011, 106, 126802</jnl>. 
$<$ lit87><jnl >N. Richter, Y.^^R. Hernandez, S. Schweitzer, J.-S. Kim, A.^^K. Patra, J. Englert,

I. Lieberwirth, A. Liscio, V. Palermo, X. Feng, A. Hirsch, K. Müllen, M. Kläui, Phys. Rev. Appl. 2017, 7, 024022</jnl >.

Table^^1 Calculated binding energies of neutral pyrene and associated functionalized variants on graphene, in their most stable AB-stacked configuration with the substituents extended and interacting with the graphene surface (Figure ${ }^{\wedge} 2\langle x$ figr 2$\left.\rangle\right) .{ }^{[a]}\langle w=3\rangle$

\begin{tabular}{|c|c|c|c|c|c|}
\hline \multirow[t]{3}{*}{ Species } & \multicolumn{3}{|c|}{ Binding energy to graphene $[\mathrm{eV}]$} & \multirow{2}{*}{\multicolumn{2}{|c|}{$\begin{array}{l}\text { Highest occupied } \\
\text { molecular orbital } \\
\text { energy }^{[\mathrm{b}]}[\mathrm{eV}]\end{array}$}} \\
\hline & \multirow{2}{*}{$\begin{array}{l}\text { Literature }^{[30]} \\
\text { (vdW-DFT) }\end{array}$} & \multicolumn{2}{|l|}{ This work } & & \\
\hline & & $\begin{array}{l}\text { Extended } \\
\text { substituents }\end{array}$ & $\begin{array}{l}\text { Non-interacting } \\
\text { substituents }^{[\mathrm{a]}]}\end{array}$ & $\begin{array}{l}\text { Isolated } \\
\text { molecule }\end{array}$ & $\begin{array}{l}\text { On } \\
\text { graphene }\end{array}$ \\
\hline Pyr & $<\mathrm{M}->1.09$ & $<\mathrm{M}->0.84$ & & $\begin{array}{l}<\mathrm{M}- \\
>5.066\end{array}$ & $\begin{array}{l}<\mathrm{M}- \\
>5.192\end{array}$ \\
\hline PAc & $<\mathrm{M}->1.35$ & $<\mathrm{M}->0.99$ & & $\begin{array}{l}<\mathrm{M}- \\
>5.379\end{array}$ & $\begin{array}{l}<\mathrm{M}- \\
>5.452\end{array}$ \\
\hline PMeAc & & $<\mathrm{M}->1.11$ & $<\mathrm{M}->0.97$ & $\begin{array}{l}<\mathrm{M}- \\
>5.051\end{array}$ & $\begin{array}{l}<\mathrm{M}- \\
>5.161\end{array}$ \\
\hline PPrAc & $<\mathrm{M}->1.54$ & $<\mathrm{M}->1.33$ & $\begin{array}{l}(<\mathrm{M}->0.95)--(<\mathrm{M}- \\
>1.01)\end{array}$ & $\begin{array}{l}<\mathrm{M}- \\
>4.969\end{array}$ & $\begin{array}{l}<\mathrm{M}- \\
>5.096\end{array}$ \\
\hline
\end{tabular}

[a] Other conformers of PMeAc and PPrAc without direct interaction with the graphene have also been explored (Figure ${ }^{\wedge \wedge} \mathrm{S} 5$, Table ${ }^{\wedge \wedge} \mathrm{S} 2$ and Table ${ }^{\wedge} \mathrm{S} 3$ ). [b] Highest occupied molecular orbital energies can be used as an indicator of oxidation potential.

Table $\wedge^{\wedge} 2$ Detailed electrochemical properties of different pyrene molecules adsorbed on MWCNT, calculated from a charge-discharge profile in Figure ${ }^{\wedge} 3<x$ figr $3>$ after subtraction of the double-layer current. $\langle w=3>$ 


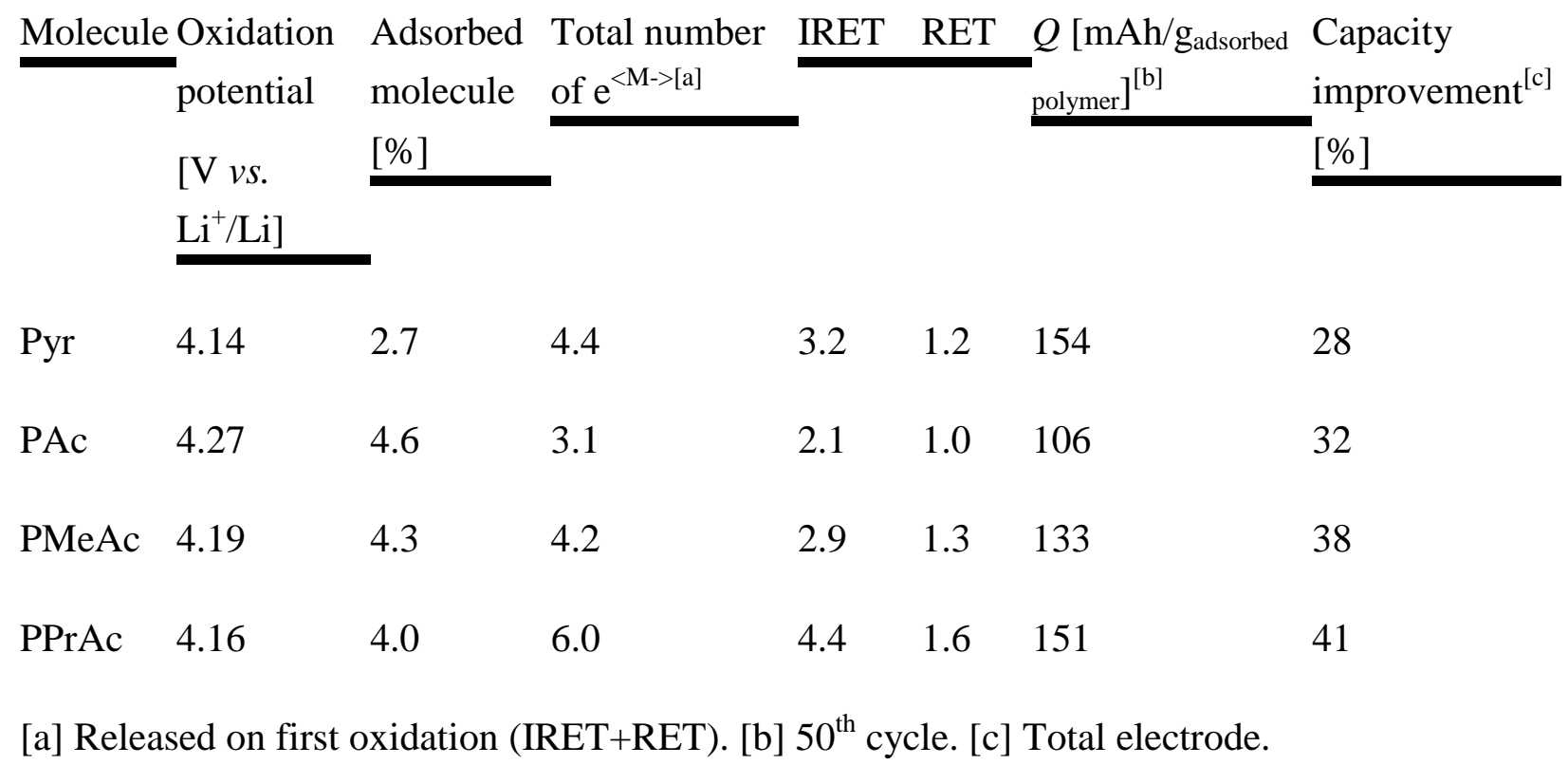

Figure $^{\wedge} 1 \quad$ a) Pyrene derivatives used in this study, b) SEM image of MWCNT along with a high-resolution image of the same sample in the inset and, c) pyrene derivative loadings per gram or per $\mathrm{cm}^{2}$ of MWCNT upon protocol 1 .

Figure $^{\wedge} 2 \quad$ a) Top and side (bottom) views of the optimized structures of the different molecules on graphene in their most stable configuration. Atom color code: red $(\mathrm{O})$, white $(\mathrm{H})$, grey (molecular C), black (graphene C).

Figure ${ }^{\wedge} 3$ a) First charge-discharge profile, b) zoomed charging part of (a), and c) $50^{\text {th }}$ charge-discharge profile of MWCNT and MWCNT adsorbed with different molecules. d) The irreversible electron transfer (IRET) on $1^{\text {st }}$ cycle and reversible electron transfer (RET) on the $50^{\text {th }}$ cycle; calculated from the capacity data in (a) on charge and (c) on discharge, respectively.

Figure $^{\wedge} 4$ Density of States (DOS) and Partial Density of States (PDOS) of graphenefunctionalized with the pyrene derivatives in their most stable configuration (binding energy indicated as $\mathrm{E}_{\mathrm{b}}$ ). Electronic levels of the isolated molecules are included for comparison. All levels have been aligned with respect to the vacuum level, defined at zero.

Figure $^{\wedge} 5$ A proposed electrochemical process of the adsorbed PMeAc on MWCNT with a possible resultant oligomer structure. The first reaction represents the oxidative 
polymerization along with corresponding proton removal (IRET). The second reversible process corresponds to the p-doping process of the resulting oligomer (RET).

Figure $^{\wedge} 6 \quad$ a) Differences in atomic Mulliken charges $(\Delta Q)$ between the cationic $(+1)$ and neutral species, represented with a color scale on the optimized structures of the cations. $b$ ) Mulliken spin distribution, expressed as number of unpaired electrons ( $\alpha-\beta$ electrons), of the cations. c) Schematic representation and nomenclature of the different chemical species, showing the numbering for the possible substitution sites of pyrene.

Figure^^7 SEM images of a) MWCNT, b) and c) CPMeAc-55 electrode surfaces at different magnification. SEM pictures of CPMeAc-55 electrode after d) $1^{\text {st }}$ e) $5^{\text {th }}$ and f) $100^{\text {th }}$ cycle.

Figure $^{\wedge \wedge} \quad$ a) Charge-discharge profile of CNTs and associated composites (Protocol 3) with different pyrene molecules at a current density of $0.1^{\wedge \wedge} \mathrm{A} / \mathrm{g}$; the last two digits of the sample name indicate the weight percentage of the molecule in that composite. b) Reversible capacity of the composite and corresponding reversible electron transfer (RET) per molecule derived from (a). c) Charge-discharge profile of MWCNT, PMeAc, and its composite (CPMeAc) according to Protocol 3, and d) capacity per gram of PMeAc and per gram of its composite CPMeAc depending on the weight percentage of molecules in that composite as derived from (c). 